\title{
An Experimental Study on the Treatment of Engineering Waste Slurry by Using Agent Conditioning-Combined Pressure Filtration
}

\author{
Yajun Wu $\mathbb{D}^{\mathbb{D}},{ }^{1}$ Rong Zhou, ${ }^{1}$ Yitian Lu $\mathbb{D}^{1},{ }^{1}$ Xudong Zhang $\mathbb{D}^{1},{ }^{1}$ and Jiansheng Lu ${ }^{2}$ \\ ${ }^{1}$ Department of Civil Engineering, Shanghai University, 99 Shangda Road, Shanghai, China \\ ${ }^{2}$ Shanghai Guanglian Environmental \& Geotechnical Engineering Co., Ltd, Baoshan District, Shanghai, China \\ Correspondence should be addressed to Yitian Lu; luyitianxxxx@shu.edu.cn and Xudong Zhang; xdzhang@shu.edu.cn
}

Received 8 January 2022; Accepted 20 January 2022; Published 14 February 2022

Academic Editor: Di Feng

Copyright (c) 2022 Yajun Wu et al. This is an open access article distributed under the Creative Commons Attribution License, which permits unrestricted use, distribution, and reproduction in any medium, provided the original work is properly cited.

Taking the waste high-moisture slurry produced at a construction site in Shanghai as the research object, the pressure filtration effect of the slurry under the condition of agent conditioning was studied. First of all, the geotechnical characteristics of the waste slurry under agent conditioning were studied through laboratory experiments, and the selection and addition amount of the agent were preliminarily determined. Then, the influence of agent addition, stirring time, and pressure filtration time on the pressure filtration efficiency was studied through field experiments. Finally, a method to guide the field filtration time by the indoor model test was put forward.

\section{Introduction}

With the rapid economic growth and the shortage of land resources, underground engineering has entered a period of rapid development. However, underground engineering involves the problem of groundwater seepage, which will lead to engineering safety problems such as foundation pit instability and tunnel collapse $[1,2]$. Therefore, it is necessary to reduce the groundwater level in the construction area through dewatering wells before the construction of underground projects. However, a large amount of highmoisture-content slurry is often produced in the process of dewatering. Construction slurry is one of the construction wastes with serious pollution and large volume and is difficult to deal with in engineering construction. In the process of construction, the waste slurry is generally treated by direct discharge, transported out of the yard through tankers, or mixed with cement. However, these treatment methods have low efficiency and high transportation costs, and they will also pollute the environment, resulting in a series of problems, such as the destruction of natural vegetation, land pollution, and river pollution. Therefore, it is necessary to deal with the construction slurry to achieve the goal of reduction, harmless, and resource utilization.
The main solid-liquid separation methods for highwater-content slurry are centrifugal dewatering, vacuum preloading, and pressure filtration dewatering [3]. However, due to the high cost and site restrictions and other reasons, centrifugal dehydration and vacuum preloading cannot effectively solve the problem of dehydration of engineering slurry. Therefore, pressure filtration dewatering is widely used in all kinds of construction sites to deal with engineering slurry. Pressure filtration is a commonly used method of solid-liquid separation, which makes the liquid seep out by applying a certain pressure to achieve the purpose of solid-liquid separation. This method has been widely used in the chemical industry, sewage treatment, waste slurry, and so on. At present, scholars have made a lot of achievements in the research of pressure filtration technology. Ruth established the mathematical background of constant-pressure filtration technology [4]. Andersen et al. and Kapur et al. proposed a method to calculate the consolidation coefficient and the degree of consolidation of the filter cake, respectively $[5,6]$. Chang et al. investigated the influence of pressure, resistance, and fluid dynamics during filtration [7-9]. Shirato and Aragaki studied the flow mechanism inside the filter cake [10]. Wakeman et al. studied the relationship between pressure, particle surface 
charge, and model parameters through experiments [11-15]. Bolton and McKinley studied the relationship among the parameters in the pressure filtration process [16]. Bezuijen et al. and Gustin et al. studied the effect of adding material and liquid viscosity on the pressure filtration effect, respectively $[17,18]$. Wu et al. studied the theory of constantpressure filtration and analyzed the formation mechanism of the filter cake [19-21]. Stamatakis and Tien explained the formation mechanism of the filter cake and gave the corresponding empirical formula $[22,23]$.

Before the pressure filtration of the waste slurry, it is necessary to use the agent to treat the slurry, and the selection and addition of the agent should be determined according to the characteristic of the waste slurry, but most of the time, due to the limitation of field conditions, it is very difficult to determine the type and dosage of the agent through the field test. Therefore, this paper puts forward a method to determine the type and dosage of the agent through the model test and gives a method to guide the field pressure filtration time by using the results of the laboratory model test. This can provide a reference for the dehydration process of waste slurry. The research process of this paper is shown in Figure 1.

\section{Laboratory Test and Materials}

2.1. Slurry Characteristic. The slurry tested is taken from the dewatering well at a construction site in Shanghai, and the physical indexes of the slurry are shown in Table 1 . As can be seen from Table 1, the slurry has high water content and poor permeability, and its $\mathrm{pH}$ value is alkaline. Figure 2 shows the particle size distribution curve of soil particles. As can be seen from Figure 2, the particle size less than $0.075 \mathrm{~mm}$ in the slurry accounted for $99.6 \%$, and the particle size less than $0.005 \mathrm{~mm}$ accounted for $36.79 \%$. The content of sand particles is relatively small, mainly composed of silt and clay.

2.2. Reagent Selection. The agent selected in this paper is quicklime and APAM. The mesh number of quicklime is 200 mesh, and the $\mathrm{CaO}$ content is more than $98 \%$. The addition amount of quicklime is added according to the mass ratio of slurry. APAM is configured as a solution with a mass ratio of $1 / 1000$ and mixed according to the volume ratio of the slurry. Quicklime can react with water to form $\mathrm{Ca}(\mathrm{OH})_{2} \cdot \mathrm{Ca}^{+}$ can have ion exchange and pozzolanic reaction with soil particles in the waste slurry, neutralize part of the negative charge on the surface, reduce the electric double layer, and increase the mutual attraction between soil particles, so small soil particles can condense into larger soil particles [24]. APAM is a kind of linear polymer, which can play the role of net capture after dissolving in water, making soil particles become flocculation groups with large diameter [25]. Both of them can increase permeability and accelerate the speed of solid-liquid separation.

After the initial selection of the reagent, we will obtain the geotechnical engineering characteristics of the slurry after the agent conditioning through the consistency test and consolidation test, then select the appropriate agent, and determine the optimal addition amount to provide the necessary parameters for the pressure filtration test.

2.3. Test Device and Procedures. After determining the use of the agent and the optimal addition amount, we will study the characteristics of the conditioning slurry under the pressure filtration condition through the pressure filtration test. After adding different mass ratios of the agent, the slurry is stirred evenly, and then the treated slurry is pumped into the plateframe filter press by the air compressor and transfer pump so that the slurry is compressed and dehydrated into cake. In the experiment, the effect of pressure filtration under different working conditions was studied by controlling the amount of the agent, stirring time, and pressure filtration time. The working conditions are shown in Table 2. The test instruments include a slurry hydrometer, pump, mixing tank, air compressor, plate and frame filter press, and electronic scale.

\section{Results and Discussion}

\subsection{Optimum Amount of the Agent}

3.1.1. Flow Characteristics. In order to study the flow characteristics of slurry after agent conditioning, consistency was used to evaluate the flow performance. The consistency of the slurry was measured by using a slurry consistency meter when the mass ratio of quicklime was $0 \%, 1 \%$, and $2 \%$ and the volume ratio was $10 \%$ APAM solution. Figure 3 shows the consistency of conditioning slurry under different conditions.

It can be seen from Figure 3 that the consistency of the original slurry relative to clear water increases slightly. After the addition of quicklime, the small particles condense into large particles with the aid of quicklime, so the slurry thickens. However, the consistency of the conditioning slurry changed little compared with the original slurry, indicating that the influence on the fluidity was small, so it was conducive to pipeline pumping and pressure filtration dehydration. After adding 1/1000 concentration of APAM solution with the volume ratio of $10 \%$, the consistency of the slurry increases obviously and is much higher than that of the original slurry, so it is not conducive to pressure filtration dewatering.

3.1.2. Characteristic of Consolidation. In order to study the dewatering consolidation performance of the conditioning slurry under different working conditions, one-dimensional compression consolidation tests were carried out to study the pressure filtration and dewatering performance of the slurry. The loading pressures were $3.125 \mathrm{kPa}, 25 \mathrm{kPa}$, $100 \mathrm{kPa}, 200 \mathrm{kPa}, 400 \mathrm{kPa}$, and $600 \mathrm{kPa}$, respectively. The working conditions are shown in Table 3.

The consolidation coefficient is a physical quantity that reflects the consolidation rate of soil under load. The larger the consolidation coefficient is, the faster the consolidation speed is, and vice versa. According to the settlement, the void 


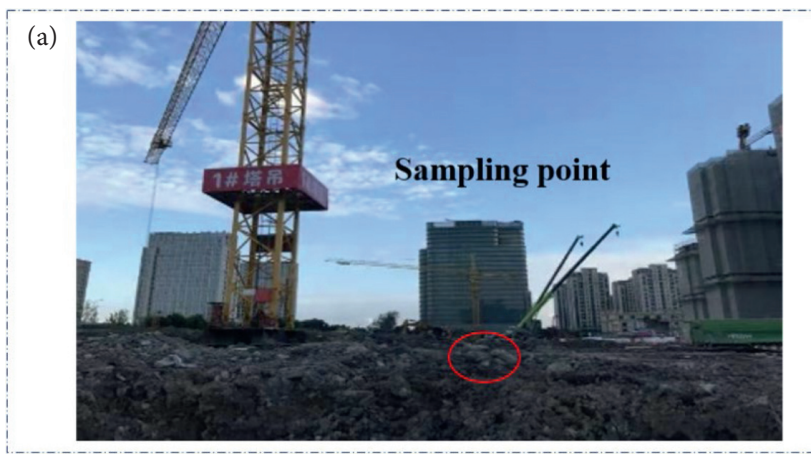

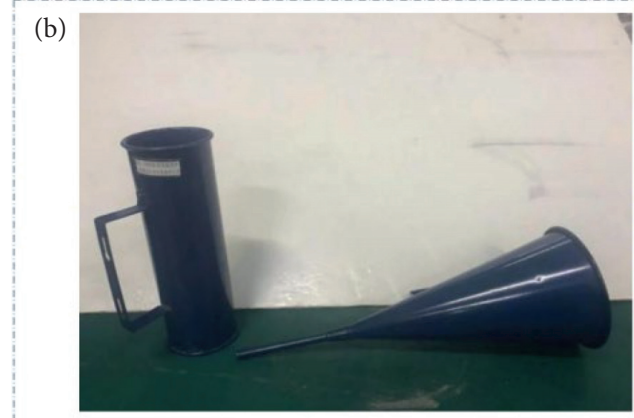

Fluidity test (c)

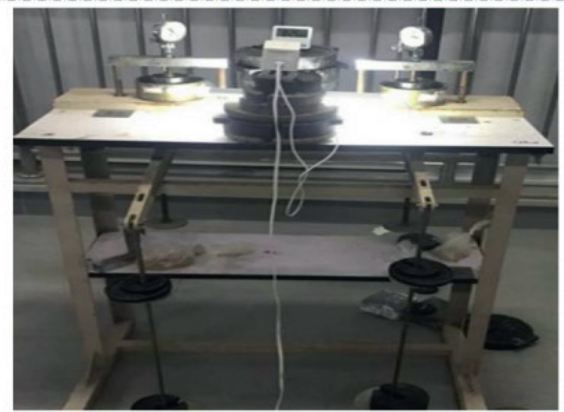

Consolidation test

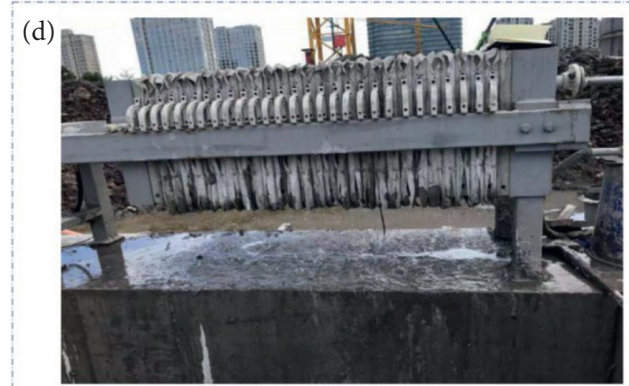

Field pressure filtration test (e)

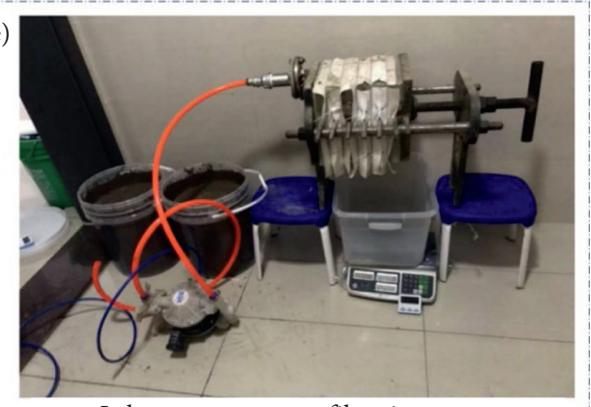

Laboratary pressure filtration test

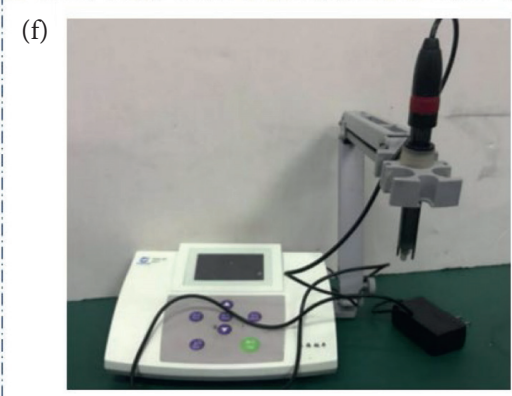

Discharged liquid treatment

Figure 1: Flow diagram of this research.

ratio under all levels of load can be obtained, the consolidation coefficient can be obtained by the time square root method, and the permeability coefficient can be calculated from the consolidation coefficient.

It can be seen from Figure 4(a) that the void ratio decreases with the increase of consolidation pressure. The void ratio of slurry after conditioning with APAM solution is smaller than that after conditioning with quicklime. This is because the flocs formed by flocculation of APAM solution are larger than those produced by flocculation of quicklime, and more supernatant is discharged at first, so the void ratio is relatively small before high-pressure compression. In 
TABle 1: Properties of slurry.

\begin{tabular}{lccc}
\hline Density, $\rho\left(\mathrm{g} / \mathrm{cm}^{3}\right)$ & Water content, $\omega(\%)$ & $\mathrm{pH}$ & Permeability coefficient $(\mathrm{m} / \mathrm{s})$ \\
\hline 1.1 & 548 & 9.2 & $2.26 \times 10^{-8}$ \\
\hline
\end{tabular}

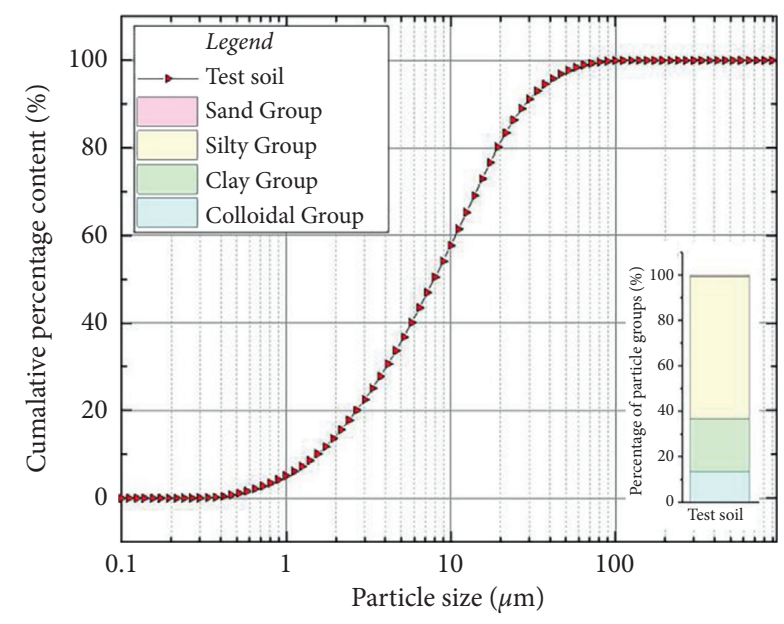

FIgURE 2: Particle size distribution.

TABle 2: Parameters under different working conditions.

\begin{tabular}{lcccc}
\hline Case & 1 & 2 & 3 & 4 \\
\hline Slurry volume $\left(\mathrm{m}^{3}\right)$ & 1.35 & 1.37 & 1.39 & 1.35 \\
Specific gravity $\left(\mathrm{g} / \mathrm{cm}^{3}\right)$ & 1.1 & 1.1 & 1.1 & 1.1 \\
Addition amount $(\%)$ & 2 & 1 & 1 & 1 \\
Mixing time $(\mathrm{min})$ & 15 & 15 & 15 & 5 \\
Constant-pressure filtration time (min) & 20 & 20 & 30 & 30 \\
\hline
\end{tabular}

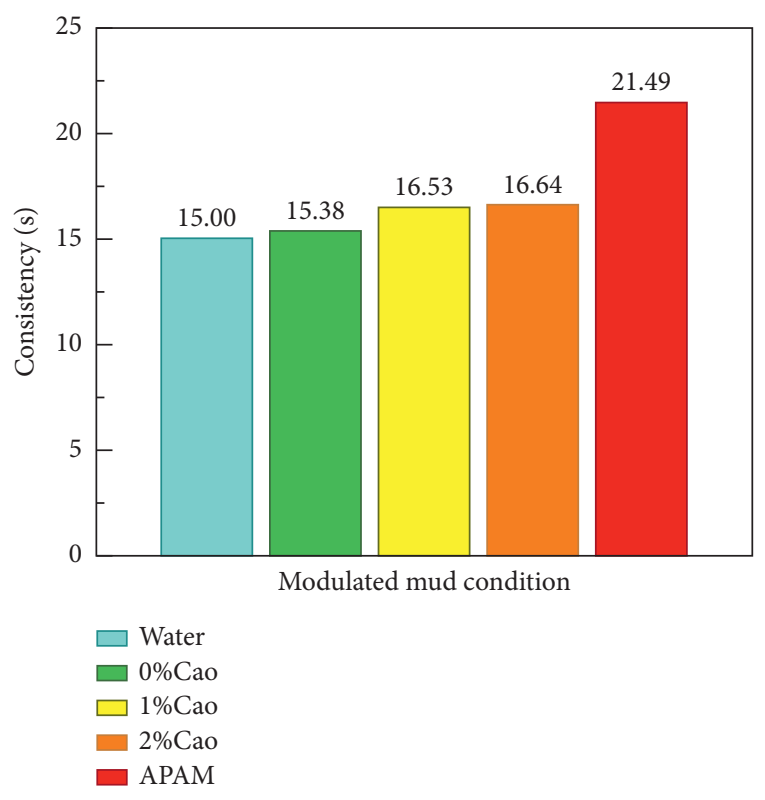

FIGURE 3: Flow performance of the slurry.

addition, it can also be obtained that the void ratio increases with the increase of the quicklime amount. This is because, in addition to flocculation, quicklime absorbs part of water and reacts with the slurry to form a large number of small
TABle 3: Addition amount of the medicament.

\begin{tabular}{lcccc}
\hline Case & 1 & 2 & 3 & 4 \\
\hline Addition methods & \multicolumn{2}{c}{ Mass percentage } & Volume percentage \\
Agents & \multicolumn{2}{c}{ Quicklime } & APAM \\
Amount & $0.5 \%$ & $1 \%$ & $2 \%$ & $10 \%$ (solution) \\
\hline
\end{tabular}

microcrystals. The more the medicament amount is, the more the crystals are formed, and these crystals continue to wrap around the soil particles, which increases the diameter of soil particles, so there is a larger gap between soil particles.

As shown in Figure 4(b), the consolidation coefficient of quicklime-treated slurry increases at first and then decreases with the increase of consolidation pressure. This is because, in the early compression process, the void ratio of treated slurry is large, no skeleton is formed between particles, and the strength is low. Under lower stress $(p<200 \mathrm{kPa})$, the pore water pressure dissipates quickly and increases with the increase of pressure. When the consolidation pressure is above $200 \mathrm{kPa}$, the soil has been compressed by the previous load, the soil void ratio decreases, the drainage channel decreases, and the pore water pressure dissipates slowly and decreases with the increase of pressure. In addition, with the increase of the amount of quicklime, the consolidation coefficient increases significantly, indicating that the increase of quicklime can accelerate the consolidation process of the slurry.

However, the consolidation coefficient of the slurry treated by APAM solution is lower than that of the slurry treated with quicklime at all levels of pressure, and the consolidation coefficient increases with the increase of pressure. This is because the consistency of the slurry conditioned by APAM solution increases greatly, and its fluidity is hindered. Under the condition of low pressure, the pore water pressure cannot dissipate quickly, and with the increase of pressure, pore water in the soil can overcome the resistance and increase the dissipation rate.

It can be seen from Figure 4(c) that the permeability coefficient decreases with the increase of consolidation pressure. When the pressure reaches $200 \mathrm{kPa}$, the change of permeability coefficient is small, and the permeability coefficients of the four groups of soil samples are of the order of $10^{-10}$ to $10^{-9} \mathrm{~m} / \mathrm{s}$. The permeability coefficient of slurry conditioned by APAM solution is lower than that of quicklime because the viscosity of slurry treated by APAM solution is high, the dissipation of pore water pressure is not smooth, and the drainage process is worse than that of quicklime. In addition, when the pressure is below $200 \mathrm{kPa}$, the permeability coefficient increases with the increase of quicklime. This is because quicklime can flocculate the small particles in the slurry into large particles and react with the slurry to form colloid, which further increases the drainage channel. Therefore, under the condition of low pressure, the increase of quicklime is conducive to the rapid dissipation of pore water pressure. 


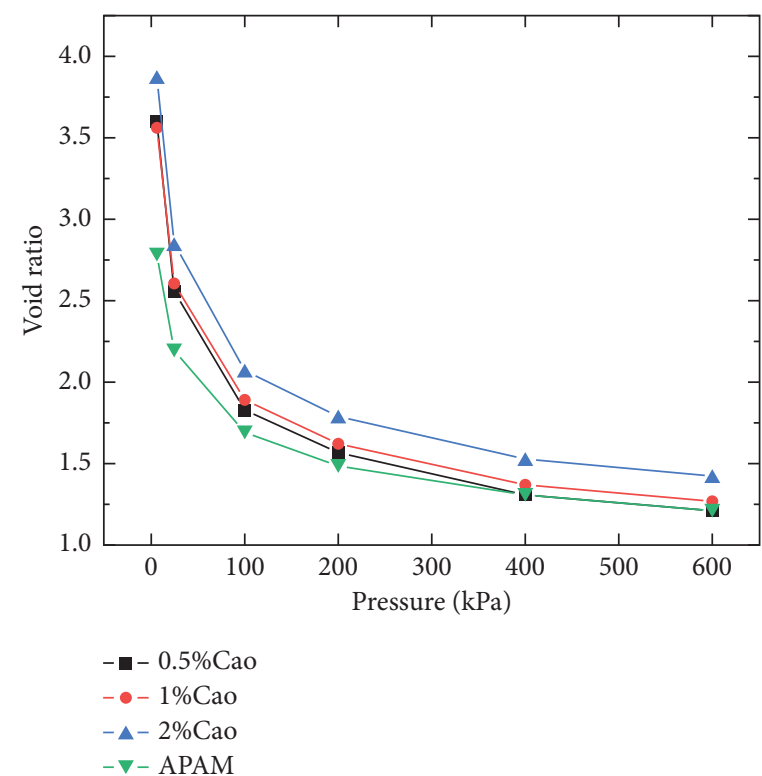

(a)

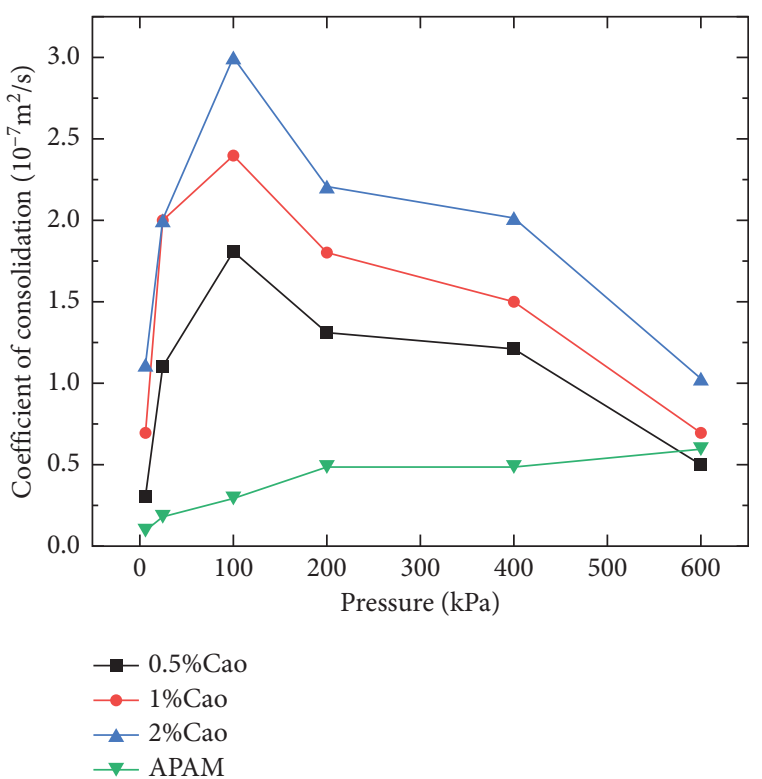

(b)

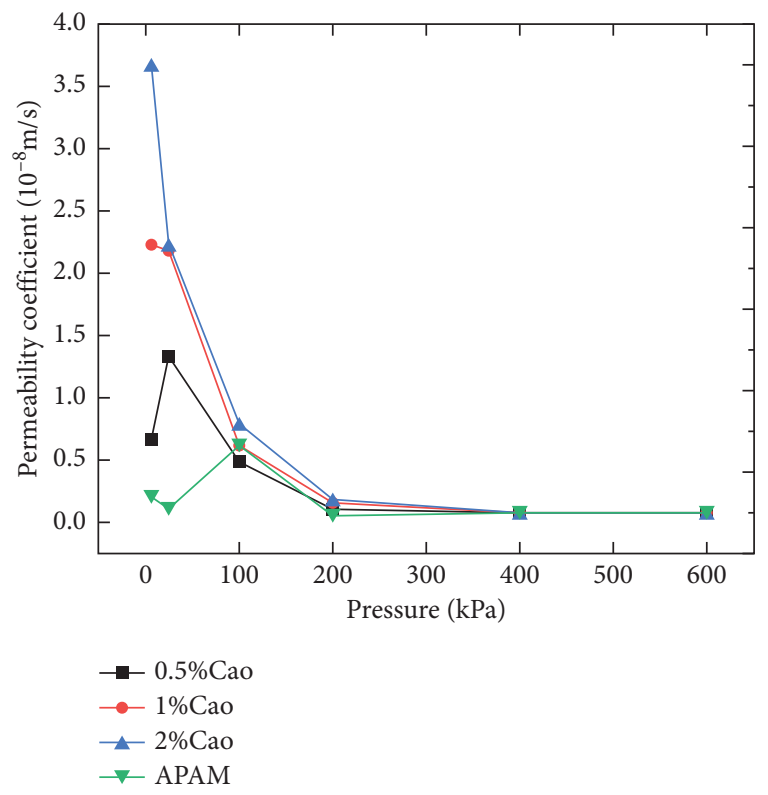

(c)

Figure 4: Slurry consolidation characteristics. (a) The $e \sim p$ relationship. (b) Curve of consolidation coefficient with pressure. (c) Curve of permeability coefficient with pressure.

Through the results of the above laboratory tests, quicklime will be selected as a suitable additive. In addition, considering the actual filtration effect and cost saving, $1 \%$ quicklime content is considered to be the optimal content. This will be applied to the field pressure filtration test for further research.

\subsection{Simulation for the Field Case}

3.2.1. Field Test Results. The distribution of the water content is shown in Figure 5.
The following can be obtained from Figure 5: (1) at the same mixing time $(15 \mathrm{~min})$ and pressure filtration time (20 $\mathrm{min}$ ), when the mass ratio of quicklime is $1 \%$ and $2 \%$, the water content of slurry cake at the same position of the filter plate is mostly close, and the average water content of the latter is $2.73 \%$ lower than that of the former, mainly because the more quicklime is added, it will absorb part of water and produce pozzolanic reactions to further consume water, further reducing the water content. (2) When the addition amount of quicklime (1\%) and the pressure filtration time (30 $\mathrm{min})$ are the same and the stirring time is $5 \mathrm{~min}$ and $15 \mathrm{~min}$, respectively, the average water content of the latter 


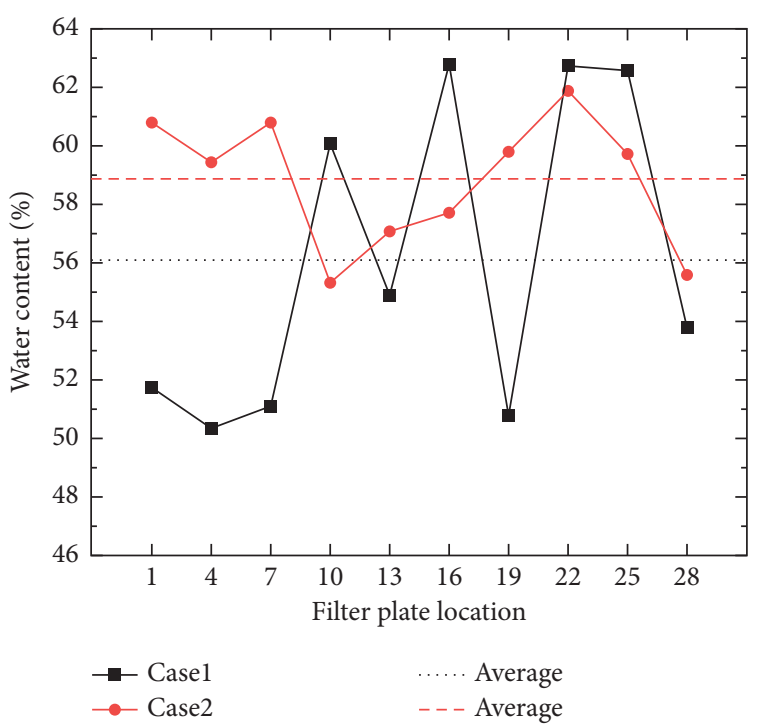

(a)

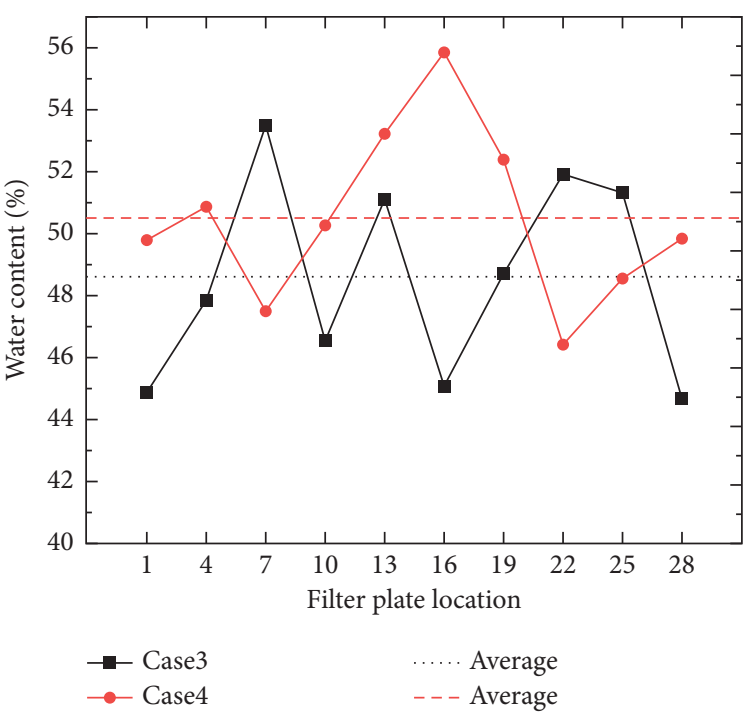

(b)

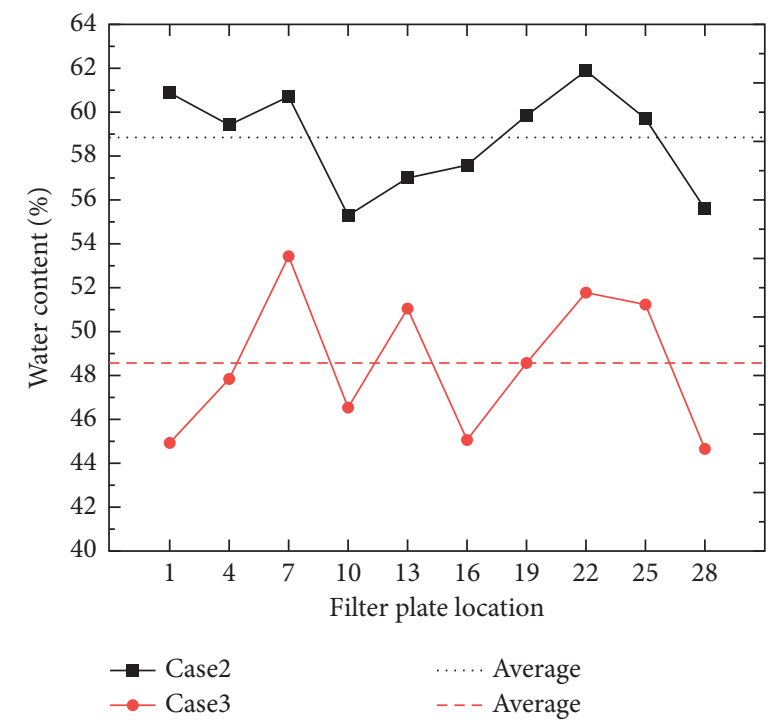

(c)

FiguRE 5: Slurry cake water content distribution under different working conditions. (a) Different addition amounts. (b) Different mixing times. (c) Different pressure filtration times.

slurry cake is $1.94 \%$ lower than that of the former. Because of the high water content and good fluidity of the slurry, quicklime can quickly dissolve in water and react with soil particles, and most of the reactions have been completed in a short time, so prolonging the stirring time cannot effectively improve the pressure filtration effect. (3) When the quicklime addition (1\%) and stirring time (15 min) are the same, the longer the pressure filtration time is, the lower the water content of the slurry cake is, and the difference in the average water content of the overall slurry cake is $10.28 \%$. This is mainly because when the filter press is filled with slurry, the slurry pressure filtration process is not only filtration but also accompanied by consolidation. The longer the consolidation time, the higher the consolidation degree of the slurry cake, so the lower the water content.
3.2.2. Prediction of Filtration Time. In this research, a method for predicting the filtration time by using the laboratory test results is proposed. On the basis of the field pressure filtration test results, the laboratory pressure filtration test was carried out under the condition of adding $1 \%$ quicklime. In this experiment, a small filter press is used to simulate the working conditions in the field. Through calculation, as shown in (1), we can obtain the filtration constant under constant pressure.

$$
V^{2}+2 V V_{e}=K A^{2} \theta
$$

where $V$ is the volume of the filtrate, $V_{e}$ is the volume of the filtrate in the filter medium, $K$ is the filtration constant, $A$ is the filtration area, and $\theta$ is the filtration time. 


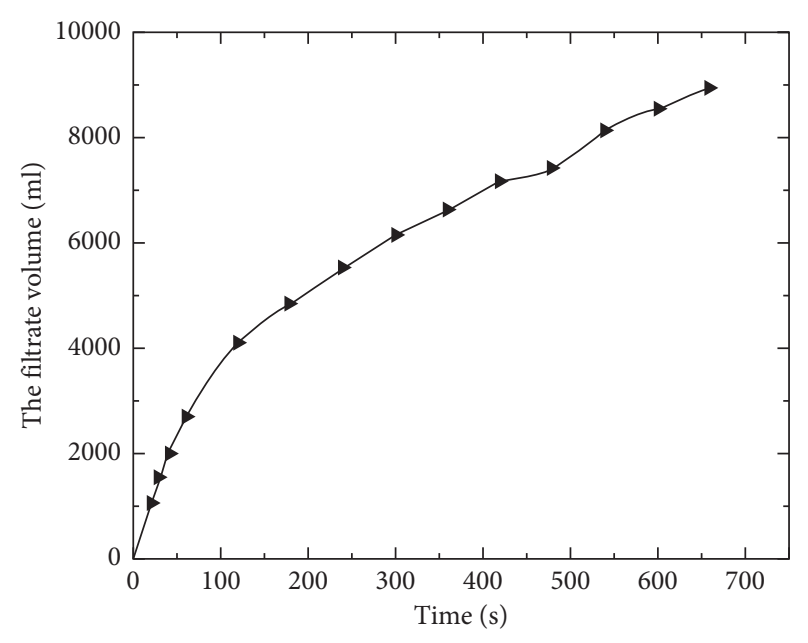

FIgURE 6: The relationship between filtrate volume and time.

Figure 6 shows the curve of the relationship between filtrate volume and time. It can be seen from the figure that the filtrate volume increases gradually with the increase of time. Through the relationship between filtrate volume and time, the value of filtration constant obtained by the constant pressure filtration formula is $5.74 \times 10^{-6} \mathrm{~m}^{2} / \mathrm{s}$. This can be used to estimate the time required for field pressure filtration.

In this section, filtration constants obtained from indoor pressure filtration will be used to estimate the time required for field pressure filtration. The basic parameters of the filter press required by calculation are as follows: the total volume of the filter chamber is $0.21 \mathrm{~m}^{3}$, the filtration area is $15.73 \mathrm{~m}^{2}$, and the volume of the filter medium is $0.012 \mathrm{~m}^{3}$. The calculation assumes that the volume of the filtrate is incompressible, so the volume of the filtrate discharged is equal to the volume change after slurry pressure filtration dehydration. The amount of the filtrate discharged can be obtained by subtracting slurry cake volume from slurry volume. Through calculation, the required time of the above four working conditions is about 16 minutes, 16 minutes, 17 minutes, and 16 minutes, respectively.

This is close to the required time of the first two conditions in the field, while the latter two conditions take longer time to obtain lower water content, indicating that the pressure filtration parameters obtained in the laboratory can be used to guide the field pressure filtration time, and the calculated pressure filtration time should be appropriately extended in order to obtain better pressure filtration effect.

3.3. Treatment for Discharged Liquid. Due to the addition of a large amount of quicklime to the slurry, the $\mathrm{pH}$ value of the waste liquid discharged by pressure filtration will increase. Measured by using the $\mathrm{pH}$ meter, the $\mathrm{pH}$ value of the waste liquid is as high as 12.3 , and the direct discharge will cause damage to the environment, so the treatment of the discharged liquid is necessary. In the experiment, citric acid and oxalic acid were used to treat the field discharged fluid to meet the discharge requirements.

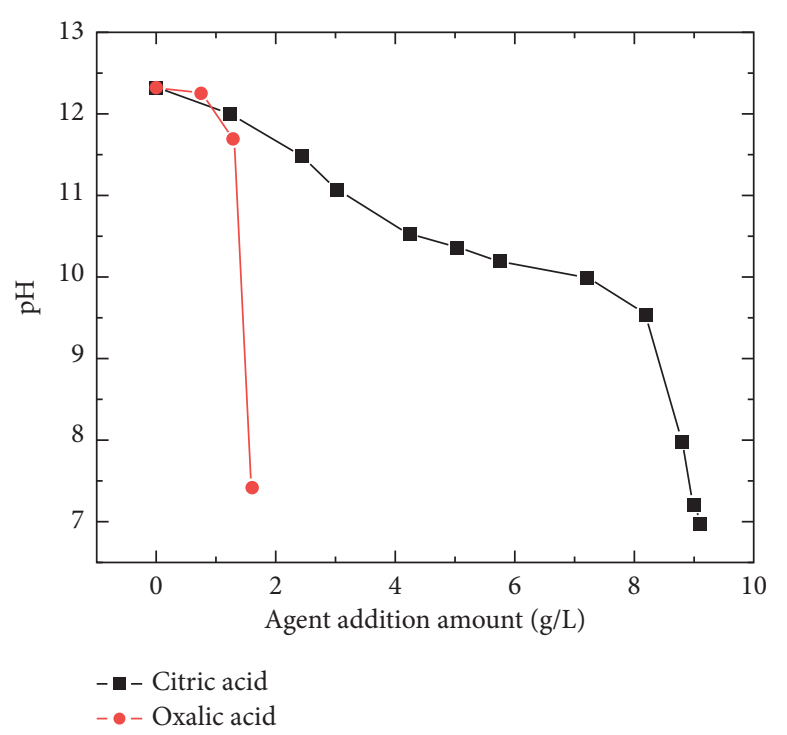

Figure 7: The relationship between the $\mathrm{pH}$ value and amount of the agent.

As can be seen from Figure 7, when the addition amount of citric acid is $8.3 \mathrm{~g}$ and oxalic acid is $1.5 \mathrm{~g}$, the $\mathrm{pH}$ value of the waste liquid can be reduced to 9 (according to the Integrated Wastewater Discharge Standard of Shanghai Municipality, the emission limit of pollutant $\mathrm{pH}$ is 6-9). Considering the large volume of slurry treatment and the high cost of direct treatment, the discharged liquid after pressure filtration will be recycled, and then unified treatment will be carried out to achieve the purpose of reducing the cost.

\section{Conclusions}

In this paper, through laboratory test and field test, the geotechnical engineering characteristics and pressure filtration effect of engineering waste slurry under different working conditions are studied, and the following conclusions can be obtained:

(1) After the slurry is conditioned by quicklime, the flocculation effect is good, and the flocculated slurry has good fluidity, which can greatly improve the solid-liquid separation rate of slurry. Considering the practical effect and cost saving, $1 \%$ is considered as the optimal amount. After the addition of APAM solution, although the slurry flocculation effect is significant, the viscosity is significantly increased, the fluidity is blocked, and consolidation and permeability are relatively small; the APAM is not suitable for pressure filtration.

(2) The pressure filtration constant calculated from the laboratory pressure filtration test can guide the required pressure filtration time in the field.

(3) After pressure filtration, the $\mathrm{pH}$ value of the discharged liquid is high, and it cannot be discharged directly. The discharged liquid can be recycled and 
neutralized in a unified way to achieve the purpose of environmental protection and cost saving.

\section{Data Availability}

The data (figures and tables) used to support the findings of this study are included within the article.

\section{Conflicts of Interest}

The authors declare that they have no conflicts of interest.

\section{Authors' Contributions}

Yajun $\mathrm{Wu}$ administered the project, provided resources, conceptualized and supervised the study, and reviewed and edited the article. Rong Zhou investigated the study, contributed to data curation, performed formal analysis, and wrote the original draft. Yitian Lu conceptualized and supervised the study, contributed to data curation, performed formal analysis, and wrote the original draft. Xudong Zhang investigated and supervised the study and wrote the original draft. Jiansheng Lu investigated the study and reviewed and edited the article.

\section{Acknowledgments}

The authors are grateful for the financial support for the study presented in this paper from the National Natural Science Foundation of China (Grant nos. 41772303 and 52108327).

\section{References}

[1] Y. Wang, Y. Liu, Y. Li, W. Jiang, and Y. Wang, "Experimental study on the failure mechanism of tunnel surrounding rock under different groundwater seepage paths," Geofluids, vol. 2021, Article ID 8856365, 2021.

[2] Z. Wei and Y. Zhu, "Seepage in water-rich loess tunnel excavating process and grouting control effect," Geofluids, vol. 2021, Article ID 5597845, 2021.

[3] Y. Wu, B. Song, Y. Lu, Q. Deng, and G. Chen, "Experimental study on the influencing factors of treatment of landfill sludge using vacuum preloading with the fenton reagent," Geofluids, vol. 2021, Article ID 9962141, 2021.

[4] B. F. Ruth, "Studies in filtration III. Derivation of general filtration equations," Industrial and Engineering Chemistry, vol. 27, no. 6, pp. 708-723, 1935.

[5] N. P. R. Andersen, M. L. Christensen, and K. Keiding, "New approach to determining consolidation coefficients using cake-filtration experiments," Powder Technology, vol. 142, no. 2-3, pp. 98-102, 2004.

[6] P. C. Kapur, S. Laha, S. Usher, R. G. deKretser, and P. Scales, "Modeling of the consolidation stage in pressure filtration of compressible cakes," Journal of Colloid and Interface Science, vol. 256, no. 1, pp. 216-222, 2002.

[7] I. L. Chang, C. P. Chu, D. J. Lee, and C. Huang, "Polymer dose effects on filtration followed by expression of clay slurries," Journal of Colloid and Interface Science, vol. 185, no. 2, pp. 335-342, 1997.
[8] K. A. Landman, L. R. White, and M. Eberl, "Pressure filtration of flocculated suspensions," AIChE Journal, vol. 41, no. 7, pp. 1687-1700, 1995.

[9] J. D. Sherwood and G. H. Meeten, "The filtration properties of compressible mud filtercakes," Journal of Petroleum Science and Engineering, vol. 18, no. 1-2, pp. 73-81, 1997.

[10] M. Shirato and T. Aragaki, "Verification of internal flow mechanism theory of cake filtration," Filtration \& Separation, vol. 9, p. 290, 1972.

[11] N. P. R. Andersen, M. L. Agerbæk, and K. Keiding, "Measurement of electrokinetics in cake filtration," Colloids and Surfaces A: Physicochemical and Engineering Aspects, vol. 213, no. 1, pp. 27-36, 2003.

[12] R. J. Wakeman, M. N. Sabri, and E. S. Tarleton, "Factors affecting the formation and properties of wet compacts," Powder Technology, vol. 65, no. 1-3, pp. 283-292, 1991.

[13] B. Yuan, Z. Li, Z. Zhao, H. Ni, Z. Su, and Z. Li, "Experimental study of displacement field of layered soils surrounding laterally loaded pile based on transparent soil," Journal of Soils and Sediments, vol. 21, no. 9, pp. 3072-3083, 2021.

[14] B. Yuan, M. Sun, Y. Wang, L. Zhai, Q. Luo, and X. Zhang, "Full 3D displacement measuring system for 3D displacement field of soil around a laterally loaded pile in transparent soil," International Journal of Geomechanics, vol. 19, no. 5, Article ID 04019028, 2019.

[15] B. Yuan, M. Sun, L. Xiong, Q. Luo, S. P. Pradhan, and H. Li, "Investigation of 3D deformation of transparent soil around a laterally loaded pile based on a hydraulic gradient model test," Journal of Building Engineering, vol. 28, Article ID 101024, 2020.

[16] M. D. Bolton and J. D. McKinley, "Geotechnical properties of fresh cement grout-pressure filtration and consolidation tests," Géotechnique, vol. 47, no. 2, pp. 347-352, 1997.

[17] A. Bezuijen, M. P. M. Sanders, and D. Den Hamer, "Parameters that influence the pressure filtration characteristics of bentonite grouts," Géotechnique, vol. 59, no. 8, pp. 717-721, 2009.

[18] E. J. G. Gustin, U. F. A. Karim, and H. J. H. Brouwers, "Bleeding characteristics for viscous cement and cementbentonite grouts," Géotechnique, vol. 57, no. 4, pp. 391-395, 2007.

[19] M. Hosseinzadeh, M. R. Mehrnia, and N. Mostoufi, "Experimental study and modeling of fouling in immersed membrane bioreactor operating in constant pressure filtration," Mathematical Problems in Engineering, vol. 2013, Article ID 456143, 2013.

[20] D. E. Smiles, "A theory of constant pressure filtration," Chemical Engineering Science, vol. 25, no. 6, pp. 985-996, 1970.

[21] Y. Wu, “An analysis of constant-pressure filtration," Chemical Engineering Science, vol. 49, no. 6, pp. 831-836, 1994.

[22] K. Stamatakis and C. Tien, "Cake formation and growth in cake filtration," Chemical Engineering Science, vol. 46, no. 8, pp. 1917-1933, 1991.

[23] C. Tien, R. Bai, and B. V. Ramarao, "Analysis of cake growth in cake filtration: effect of fine particle retention," AIChE Journal, vol. 43, no. 1, pp. 33-44, 1997.

[24] P. N. Johnson and A. Amirtharajah, "Ferric chloride and alum as single and dual coagulants," Journal of the American Water Works Association, vol. 75, no. 5, pp. 232-239, 1983.

[25] S. K. Dentel and J. M. Gossett, "Mechanisms of coagulation with aluminum salts," Journal of the American Water Works Association, vol. 80, no. 4, pp. 187-198, 1988. 